\title{
Generalized Plücker Formulae
}

By

Tohsuke URABE*

\section{Contents}

§. Introduction

$\S 1$. Preliminaries

347

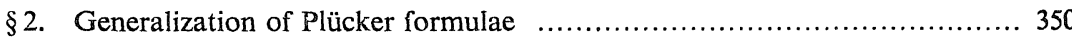

§. Examples

356

\section{§0. Inmtroductiom}

Since Poncelet discovered the so-called Plücker formulae for plane algebraic curves, many attempts to generalize them have been made by many mathematicians.

Recently $R$. Piene in her papers [4] and [5] reviewed the work on generalizing Plücker formulae by Weyl, Pohl, Teissier and others, and obtained further interesting results. In [5] she found that the problem was reduced to representing the polar classes by intrinsic quantities in the Chow group, and showed the formulae in restricted cases.

Following her idea and stimulated by the author's result on duality of polar classes [8], the author obtained some formulae in several different cases from hers. The aim of this article is to formulate them.

Let $X$ be an $r$-dimensional algebraic variety and $f: X \rightarrow \mathbb{P}^{n}$ be a generic immersion to a projective space of dimension $n$. For every integer $k$ with $0 \leqq k \leqq r$, the $k$-th polar class $\left[M_{k}\right] \in A_{r-k} X$ is defined in the Chow homology group A.X. (See [2], [3] and [4].)

Satisfactory formulae for $\left[M_{1}\right]$ have been obtained by Pohl and Piene ([6], [5]). Pohl's formula treats the case where $X$ is smooth, while Piene's

Conmunicated by S. Nakano, May 2, 1979.

* Research Institute for Mathematical Sciences, Kyoto University, Kyoto 606, Japan. Present address: Department of Mathematics, Tokyo Metropolitan University, Tokyo 158 , Japan. 
one treats the case where $f$ is an embedding. Moreover in the case where $\operatorname{dim} X=n-1$, we can obtain formulae for every $\left[M_{k}\right]$ by slight modification of the deduction with respect to $\left[M_{1}\right]$.

The main cases discussed in this article are the following;

I. $f$ is an embedding. $X$ is a local complete intersection. And the conormal bundle $N$ of $X$ in $\boldsymbol{P}^{n}$ is a successive extension by line bundles. (These conditions are satisfied if $X$ is a complete intersection in $\boldsymbol{P}^{n}$.)

II. $X$ is smooth. The cotangent bundle $\Omega_{X}$ of $X$ is a successive extension by line bundles.

We will give explicit formulae for every $\left[M_{k}\right]$ with $0 \leqq k \leqq r$. (Theorem 2.2, Theorem 2.3 and Theorem 2.4.)

As a matter of fact, we can manipulate more general cases where $N$ or $\Omega_{X}$ has a filtration by subbundles and the Fitting ideals associated with the filtration satisfy an additional condition. (See Theorem 2.2 and Theorem 2.3.) In this last version our main theorems give not only the formulae for the cases I, II, but also those for the classical case where $X$ is a smooth subvariety of $\boldsymbol{P}^{n}$. In the classical case the polar class $\left[M_{k}\right]$ coincides with the Todd's canonical classes, which are discussed in Porteous [7].

Section 1 is devoted to preliminaries. Section 2 is the main part of this article. Associated with a filtration of the conormal bundle or the cotangent bundle of the variety $X$, we define a sequence of ideals. Let $\pi: \bar{X} \rightarrow X$ be the blowing up of these ideals. We construct on $\bar{X}$ a vector bundle $P$ which is a modification of $P_{X}(L)$, the sheaf of principal parts of order 1 . Then, comparing the Chern classes, we obtain the desired formulae. In the last section Section 3, we apply the obtained formulae for several examples.

The author would like to express his hearty thanks to Professor B. Teissier for his interesting lectures during his stay at the R.I.M.S. By him the author was first taught a formula which is generalized in this article.

\section{§1. Preliminaries}

We first fix the notation to be used throughout this article.

$X$ : a projective variety i.e. a reduced (possibly reducible and singular) equidimensional projective scheme over an algebraically closed ground field $k$ of arbitrary characteristic.

$r$ : the dimension of $X$. 
$\boldsymbol{P}^{n}$ : a projective space of dimension $n$.

$f: X \rightarrow \mathbb{P}^{n}$ : a generic immersion i.e. a morphism from $X$ to $\mathbb{P}^{n}$ of which the restriction to an open dense subset of $X$ is an immersion.

$\mathcal{O}_{Z}$ : the structure sheaf of a scheme $Z$.

$\Omega_{Z}$ : the sheaf of differentials on $Z$. It is called the cotangent bundle if $Z$ is smooth.

$\Omega_{g}$ : the sheaf of relative differentials with respect to a morphism $g$.

$V=s_{P^{n *}} \mathcal{O}_{\mathbb{P}^{n}}(1)$

$V_{Z}=s_{Z}^{*} V$

Here $s_{Z}$ denotes the structure morphism $s_{Z}: Z \rightarrow \operatorname{Spec}(k)$ of a scheme $Z$. Note that $\operatorname{dim}_{k} V=n+1$.

$L=f^{*} \mathcal{O}_{P^{n}}(1):$ the pull-back of the hyperplane line bundle to $X$.

$P_{X}(L)$ : the sheaf of principal parts of order 1 of $L$.

a: $\quad V_{X} \rightarrow P_{X}(L)$ : the canonical morphism.

As for $P_{X}(L)$ and the morphism a, see the appendix of Piene [4].

A.Z: the Chow homology group of a scheme $Z$.

$\mathrm{A}^{\circ} Z$ : the Chow cohomology ring of a projective scheme $Z$. (See Fulton [2].)

We recall that there exist exact sequences of sheaves (Piene [5])

$$
\begin{aligned}
& V_{X} \stackrel{a}{\longrightarrow} P_{X}(L) \longrightarrow \Omega_{f} \otimes L \longrightarrow 0 \\
& 0 \longrightarrow \Omega_{X} \otimes L \longrightarrow P_{X}(L) \longrightarrow L \longrightarrow 0 .
\end{aligned}
$$

If $X$ is a local complete intersection in $\mathbb{P}^{n}$, the sequence

$$
0 \longrightarrow N \otimes L \longrightarrow V_{X} \stackrel{a}{\longrightarrow} P_{X}(L) \longrightarrow 0
$$

is also exact, where $N$ denotes the conormal bundle of $X$.

$F^{i}(M)$ denotes the $i$-th Fitting ideal of a coherent sheaf $M$ on a Noetherian scheme $Z$, i.e. the sheaf of ideals generated at each point $z \in Z$ by the $(q-i)$ minors of the matrix of any presentation

$$
\mathcal{O}_{Z, z}^{p} \longrightarrow \mathcal{O}_{Z, z}^{q} \longrightarrow M_{z} \longrightarrow 0 \text {. }
$$

We say "a vector bundle" instead of "a locally free coherent sheaf". For two vector bundles $M^{\prime}, M$ such that $M^{\prime}$ is a locally free subsheaf of $M$, we say particularly that $M^{\prime}$ is a subbundle of $M$ if the residue $M / M^{\prime}$ is also a vector bundle.

Next propositions are fundamental in what follows.

On the definition of the polar class $\left[M_{k}\right] \in A_{r-k} X$, we refer readers to Piene [5] and the author's [8]. 
Proposition 1.1. If $\pi: Z \rightarrow X$ is any proper birational morphism such that the surjective morphism of vector bundles $\left.a\right|_{U}:\left.V_{U} \rightarrow P_{X}(L)\right|_{U}$ extends to a surjective morphism of vector bundles $b: V_{Z} \rightarrow P$, there is an equality in $A . X$

$$
\left[M_{k}\right]=\pi_{*}\left(c_{k}(P) \cap[Z]\right)
$$

for every $k$, where $U$ denotes the maximal smooth open subscheme of $X$ on which $f$ is an immersion.

For the proof see Piene [5].

Lemma 1.2. Let $\alpha: E \rightarrow F$ be a homomorphism of bundles on a scheme $Y$, of ranks $n$ and $m$, and put $I=F^{0}(\operatorname{Coker}(\alpha))$. Then $I$ is invertible if and only if $\operatorname{Im}(\alpha)$ is an $m$-bundle.

This is Lemma (1.1) in Piene [5].

\section{§2. Generalization of Plücker Formulae}

Recall that $f: X \rightarrow \mathbb{P}^{n}$ denotes a generic immersion.

In this section we will give formulae for every polar class $\left[M_{k}\right] \in A . X$ with respect to $f$ in several cases.

A vector bundle $M$ on $X$ is said to be $a$ successive extension by line bundles if there exists a sequence of surjective morphism of vector bundles

$$
M=M_{m} \stackrel{\alpha_{m-1}}{\longrightarrow} M_{m-1} \stackrel{\sigma_{m-2}}{\longrightarrow} \cdots \stackrel{\alpha_{2}}{\longrightarrow} M_{2} \stackrel{\alpha_{1}}{\longrightarrow} M_{1}
$$

with $\operatorname{rank} M_{i}=i$. This condition is equivalent to saying that the structure group of $M$ can be reduced to the group of upper-semi-triangular matrices.

Case I. The morphism $f$ is an embedding. $X$ is a local complete intersection. And the conormal bundle $N$ of $X$ in $\mathbb{P}^{n}$ is a successive extension by line bundles.

Let $K=\operatorname{Ker}\left(a: V_{X} \rightarrow P_{X}(L)\right)$. By assumption we know that $K$ is a vector bundle and it is isomorphic to $N \otimes L$. Associated with the ascending sequence of subbundles of $N$, there exists a sequence of subbundles

$$
0=K_{0} \subset K_{1} \subset K_{2} \subset \cdots \subset K_{n-r}=K
$$

with $\operatorname{rank} K_{i}=i$. Set $F_{i}=F^{n+1-i}\left(V_{X} / K_{i}\right)$. In other words we define $F_{i}$ to be the sheaf of ideals which is locally generated by all maximal minors of the matrix which represents the inclusion $K_{i} \rightarrow V_{X}$ on some open set. 
For every point $x \in X$, we choose a sufficiently small neighbourhood $U$ of $x$ and fix a local trivialization such that the next diagram is commutative for every integer $i$ with $1 \leqq i \leqq n-r$,

$$
\begin{gathered}
\left.K\right|_{U} \cong \mathcal{O}_{U}^{n-r} \\
\hat{\jmath} \hat{\jmath}^{i} \\
\left.K_{i}\right|_{U} \cong \mathcal{O}_{U}^{i}
\end{gathered}
$$

where $\mathcal{O}_{U}^{i} \hookrightarrow \mathcal{O}_{U}^{n-r}$ denotes the inclusion into the product of first $i$-factors. By fixing a base of $V$, we also obtain an induced isomorphism $V_{X} \cong \mathcal{O}_{X}^{n+1}$. Let $\mathfrak{I}=\{U\}$ be a covering of $X$ whose each member has the above property. Let $\left\{g_{U r}\right\}_{U, V \in \mathbb{N}}$ be the transition functions of the vector bundle $K$ associated with the above datum. Then, every $g_{U V}$ is represented by an upper-semi-triangular matrix. Representing the inclusion

$$
\left.\mathcal{O}_{U}^{n-r} \cong K\right|_{U} \longleftrightarrow V_{U} \cong \mathcal{O}_{U}^{n+1}
$$

by a matrix

$$
R=\left(r_{i j}\right)_{0 \leqq i \leqq n, 1 \leqq j \leqq n-r}
$$

we know that $F_{1}$ is the ideal generated by the elements in the first column, $F_{2}$ is the ideal generated by the minors of degree 2 of the first and second columns,..., and $F_{n-r}$ is the ideal generated by the maximal minors of the above matrix $R$. We obtain the descending sequence of sheaves of ideals

$$
F_{1} \supseteq F_{2} \supseteq \cdots \supseteqq F_{n-r} .
$$

Since $F_{n-r}=F^{r+1}\left(P_{X}(L)\right)=F^{r}\left(\Omega_{X}\right), F_{n-r}$ is nothing but the Jacobian ideal of $X$.

Definition 2.1. For a sequence of sheaves of ideals on $X$

$$
I_{1} \supseteqq I_{2} \supseteqq \cdots \supseteqq I_{p}
$$

we define the successive blowing up associated with (I) $\pi: \bar{X} \rightarrow X$ inductively as follows; if $p=1, \pi$ is the blowing up of the ideal $I_{1}$; if $p>1, \pi$ is the composition of $\pi_{1}$ with the blowing up of the ideal $I_{p} \mathcal{O}_{X_{1}}$ where $\pi_{1}: \bar{X}_{1} \rightarrow X$ denotes the successive blowing up associated with the sequence $I_{1} \supseteqq I_{2} \supseteqq \cdots \supseteqq I_{p-1}$.

By definition every ideal $I_{j} \mathcal{O}_{X}$ with $1 \leqq j \leqq p$ is locally principal. We note that the sheaf of ideals

$$
\mathscr{H}_{\text {and } \bar{X}}\left(I_{j-1} \mathcal{O}_{X}, I_{j} \mathcal{O}_{X}\right) \hookrightarrow \mathscr{H}_{a m_{\bar{X}}}\left(I_{j-1} \mathcal{O}_{\bar{X}}, I_{j-1} \mathcal{O}_{\bar{X}}\right)=\mathcal{O}_{X}
$$

is also locally principal for $j>1$.

Remark. Indeed we can easily observe that $\pi: \bar{X} \rightarrow X$ is equal to the blowing 
up of the product ideal $I_{1} \cdot I_{2} \cdots \cdot I_{p}$.

Let $\pi: \bar{X} \rightarrow X$ be the successive blowing up associated with (F). We note that

$$
0 \longrightarrow \pi^{*} K \longrightarrow V_{\bar{X}} \longrightarrow \pi^{*} P_{X}(L) \longrightarrow 0
$$

is still exact, since injectivity of $\pi^{*} K \rightarrow V_{\bar{X}}$ at the generic point of $\bar{X}$ induces injectivity everywhere on $\bar{X}$ because $\pi^{*} K$ is torsion free. We denote a sufficiently fine refinement of the covering $\pi^{-1 \mathfrak{U}}=\left\{\pi^{-1} U\right\}$ with the properties described below again by $\mathfrak{U}=\{U\}$. Set $K^{(0)}=\pi^{*} K$. We concentrate our attension on an arbitrary $U \in \mathfrak{U}$. By abuse of notation let the inclusion

$$
\left.\mathcal{O}_{U}^{n-r} \cong K^{(0)}\right|_{U} \longrightarrow V_{U} \cong \mathcal{O}_{U}^{n+1}
$$

be represented also by the matrix $R$. We set $\bar{F}_{i}=\mathscr{H}_{a m_{0} \bar{x}}\left(F_{i-1} \mathcal{O}_{\bar{X}}, F_{i} \mathcal{O}_{X}\right)$ for $1 \leqq i \leqq n-r$. It may be assumed that $\left.\bar{F}_{i}\right|_{U}$ is generated by one element in $\Gamma\left(U, \mathcal{O}_{\bar{X}}\right)$ for every integer $i$. Let $s_{U} \in \Gamma\left(U, \mathcal{O}_{\bar{X}}\right)$ be a generator of $\bar{F}_{1} l_{U}$ $=\left.F_{1} \mathcal{O}_{X}\right|_{U}$. We can write that $r_{i 1}=r_{i 1}^{\prime} s_{U}$ with $r_{i 1}^{\prime} \in \Gamma\left(U, \mathcal{O}_{\bar{X}}\right)$ for every integer $i$ with $0 \leqq i \leqq n$. There exists an integer $i_{1}$ such that $r_{i_{1} 1}^{\prime}$ is an invertible element in $\Gamma\left(U, \mathcal{O}_{X}\right)$. We define a locally free sheaf $K^{(1)}$ by the transition functions $\left\{g_{U V}^{(1)}\right\}_{U, V \in \mathfrak{u}}$ with

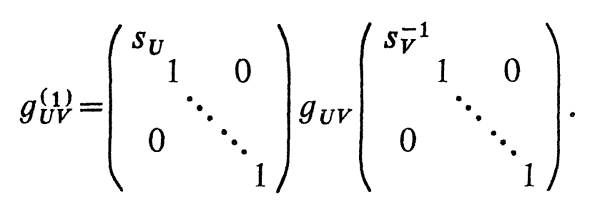

It is easy to check that every entry in $g_{U V}^{(1)}$ belongs to $\Gamma\left(U \cap V, \mathcal{O}_{X}\right)$, when we note that $g_{U V}$ is an upper-semi-triangular matrix. $K^{(1)}$ is the locally free subsheaf of $V_{X}$ generated on $U$ by the column vectors of

$$
R_{1}^{\prime}=\left(r_{i 1}^{\prime}, r_{i 2}, \ldots, r_{i, n-r}\right)_{0 \leqq i \leqq n} .
$$

By appropriate change of local trivialization of $K^{(0)}$ and $K^{(1)}$ compatible with their filtration, we assume $R_{1}^{\prime}$ is, from the beginning, of the form that $r_{i_{1}, 1}^{\prime}=1$ and $r_{i_{1}, j}=0$ for $j>1$. Under this presentation $\bar{F}_{2}$ is nothing but the ideal generated on $U$ by the entries in the second column of $R_{1}^{\prime}$. There exists a commutative exact diagram of sheaves 


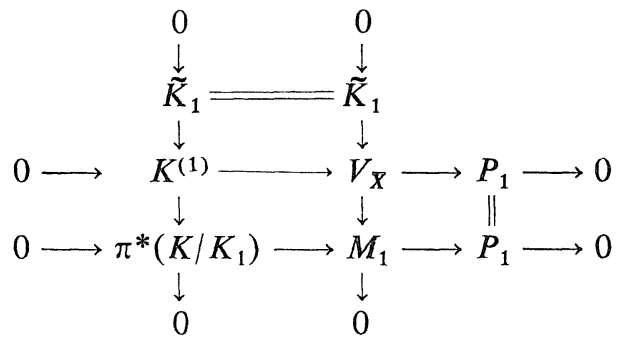

where $\tilde{K}_{1}=\pi^{*} K_{1} \otimes \bar{F}_{1}^{-1}, M_{1}=V_{\bar{X}} / \tilde{K}_{1}$ and $P_{1}=V_{\bar{X}} / K^{(1)}$.

Since the inclusion $\widetilde{K}_{1} \rightarrow V_{X}$ is represented by the first column of $R_{1}^{\prime}$ and $r_{i_{1}, 1}^{\prime}=1$, we notice that $M_{1}$ is a vector bundle. The inclusion $\pi^{*}\left(K / K_{1}\right) \rightarrow M_{1}$ is represented by the matrix $R_{2}$ which is obtained by eliminating the first column and the $i_{1}$-th row from $R_{1}^{\prime}$. In the Chow ring $\mathrm{A}^{\cdot} \bar{X}$ an equality of Chern classes

$$
c\left(M_{1}\right)=c\left(\pi^{*} K_{1} \otimes \bar{F}_{1}^{-1}\right)^{-1}
$$

holds.

Let $t_{U} \in \Gamma\left(U, \mathcal{O}_{\bar{X}}\right)$ be a generator of $\left.\bar{F}_{2}\right|_{U}$. We can write that $r_{i 2}=r_{i 2}^{\prime} t_{U}$ with $r_{i 2}^{\prime} \in \Gamma\left(U, \mathcal{O}_{\bar{X}}\right)$ for every integer $i$ with $0 \leqq i \leqq n$ and there exists $i_{2}$ different from $i_{1}$ such that $r_{i_{2}, 2}^{\prime}$ is invertible. We define a locally free sheaf $K^{(2)}$ by the transition functions $\left\{g_{U V}^{(2)}\right\}$ with

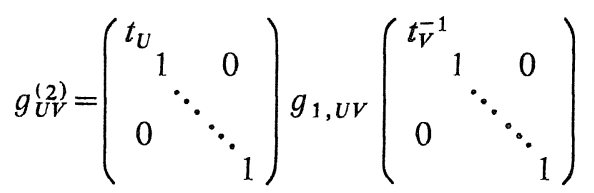

where $\left\{g_{1, U V}\right\}$ denotes the system of transition functions of the bundle $\pi^{*}\left(K / K_{1}\right)$, which is obtained by eliminating the first column and the first row from $\left\{g_{U V}\right\}$. Let $R_{2}^{\prime}$ be the matrix obtained by dividing every entries in the first column of $R_{2}$ by $t_{U}$. $\quad R_{2}^{\prime}$ represents a injective morphism $K^{(2)} \rightarrow M_{1}$. We have a commutative exact diagram of sheaves

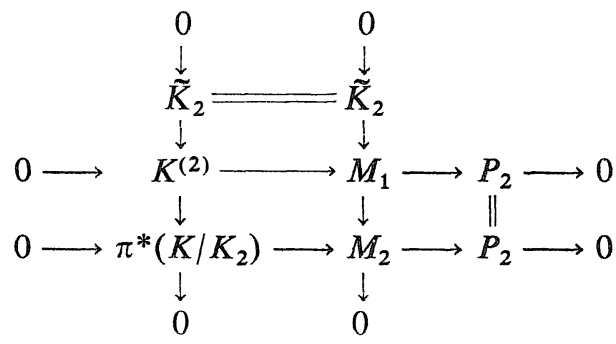


where $\widetilde{K}_{2}=\pi^{*}\left(K_{2} / K_{1}\right) \otimes \bar{F}_{2}^{-1}, M_{2}=M_{1} / \widetilde{K}_{2}$ and $P_{2}=M_{1} / K^{(2)}$, and in the Chow ring $c\left(M_{2}\right)=c\left(M_{1}\right) c\left(\pi^{*}\left(K_{2} / K_{1}\right) \otimes \bar{F}_{2}^{-1}\right)^{-1}$ holds.

Repeating this process $(n-r)$-times we obtain at last a vector bundle $P=P_{n-r} \cong M_{n-r}$ and a surjective morphism $V_{X} \rightarrow P$ which is the composition of $V_{\bar{X}} \rightarrow M_{1}, M_{1} \rightarrow M_{2}, \ldots$, and $M_{n-r-1} \rightarrow M_{n-r} \cong P_{n-r}=P$. Moreover

$$
c(P)=c\left(\pi^{*} K_{1} \otimes \bar{F}_{1}^{-1}\right)^{-1} c\left(\pi^{*}\left(K_{2} / K_{1}\right) \otimes \bar{F}_{2}^{-1}\right)^{-1} \ldots c\left(\pi^{*}\left(K_{n-r} / K_{n-r-1}\right) \otimes \bar{F}_{n-r}^{-1}\right)^{-1}
$$

holds in $A^{\circ} \bar{X}$.

Obviously by the above construction the surjective morphism of vector bundles $V_{X} \rightarrow P$ extends the morphism $\left.a\right|_{X_{s}}:\left.V_{X_{s}} \rightarrow P_{X}(L)\right|_{X_{s}}$ where $X_{s}$ denotes the maximal smooth open subscheme of $X$.

Reviewing the above proof we notice that if $\bar{F}_{i}=\mathcal{O}_{X}$ we can omit the $i$-th step and then we need not assume that $\operatorname{rank}\left(K_{i} / K_{i-1}\right)=1$. Moreover we can formulate the theorem even if for a birational morphism $\rho: X^{\prime} \rightarrow X, \rho^{*} N$ has such a filtration. We know that Hironaka-Kleiman's theorem assures the existence of a proper birational morphism $\rho: X^{\prime} \rightarrow X$ such that $\rho^{*} N$ is a successive extension by line bundles for any local complete intersection $X$.

Refering to Proposition 1.1, we obtain the next theorem.

Theorem 2.2. Let $X \subset \mathbb{P}^{n}$ be a local complete intersection of dimension $r$ in a projective $n$-space, and $\rho: X^{\prime} \rightarrow X$ be a birational proper morphism. Let

$$
0=N_{0} \subset N_{1} \subset N_{2} \subset \cdots \subset N_{p}=\rho^{*} N
$$

be a filtration by subbundles on $X^{\prime}$. There exists canonical injective morphism $N_{i} \otimes \rho^{*} L \rightarrow V_{X^{\prime}}$ for every i. Set $F_{i}=F^{n+1-\operatorname{rank}\left(N_{i}\right)}\left(V_{X^{\prime}} / N_{i} \otimes \rho^{*} L\right)$ i.e. the Fitting ideal of maximal rank for every $i \geqq 0$. Assume that $F_{i}=F_{i-1}$ if $\operatorname{rank}\left(N_{i} / N_{i-1}\right)>1$. Let $\bar{\pi}: \bar{X} \rightarrow X^{\prime}$ be the successive blowing up associated with the sequence of ideals $F_{1} \supseteqq F_{2} \supseteqq \cdots \supseteqq F_{p}$. Set $\pi=\rho \bar{\pi}$ and $\bar{F}_{i}$ $=\mathscr{H}_{a m_{0}}\left(F_{i-1} \mathcal{O}_{\bar{X}}, F_{i} \mathcal{O}_{\bar{X}}\right)$ for every $i$. Then, every $\bar{F}_{i}$ is a locally principal sheaf of ideals and the polar class $\left[M_{k}\right] \in A_{r-k} X$ satisfies following equalities

$$
\begin{aligned}
c(P) & =\sum_{i} c_{i}(P) \\
& =c\left(Q_{1}\right)^{-1} c\left(Q_{2}\right)^{-1} \cdots c\left(Q_{p}\right)^{-1}
\end{aligned}
$$

where $Q_{i}=\bar{\pi}^{*}\left(\left(N_{i} / N_{i-1}\right) \otimes \rho^{*} L\right) \otimes \bar{F}_{i}^{-1}$ for $1 \leqq i \leqq p$, and

$$
\left[M_{k}\right]=\pi_{*}\left(c_{k}(P) \cap[\bar{X}]\right) \quad \text { for every } k \geqq 0 \text {. }
$$

Case II. $X$ is smooth. The cotangent bundle $\Omega_{X}$ of $X$ is a successive extension by line bundles. 
The proof and the result are the dual version of those in Case I. Therefore we only give an outline of the proof.

Let

$$
0=\Omega_{r} \subset \Omega_{r-1} \subset \cdots \subset \Omega_{2} \subset \Omega_{1} \subset \Omega_{0}=\Omega_{X}
$$

be the associated filtration by subbundles with rank $\Omega_{i}=r-i$. Let $F_{i}=$ $F^{0}\left(\operatorname{Cokcr} \phi_{i}\right)$ where $\phi_{i}: f^{*} \Omega_{\mathbb{p}^{n}} \otimes L \rightarrow\left(\Omega_{X} / \Omega_{i}\right) \otimes L$ denotes the composition of the canonical morphism $f^{*} \Omega_{p^{n}} \rightarrow \Omega_{X} \otimes L$ with the projection $\Omega_{X} \otimes L \rightarrow\left(\Omega_{X} / \Omega_{i}\right) \otimes L$. $F_{i}$ is the ideal generated by the maximal minors of the representation of $\phi_{i}$ by a matrix. Recall that $F_{r}$ is equal to the ramification ideal of the morphism $f$.

Let $\pi: \bar{X} \rightarrow X$ be the successive blowing up associated with the sequence $F_{1} \supseteqq F_{2} \supseteqq \cdots \supseteqq F_{r} . \quad$ Set $\bar{F}_{i}=\mathscr{H}_{a m^{\prime} \bar{X}}\left(F_{i-1} \mathcal{O}_{X}, F_{i} \mathcal{O}_{\bar{X}}\right)$ for $i \geqq 1$. Let $P$ be the image of $\pi^{*}(a): V_{\bar{X}} \rightarrow \pi^{*} P_{X}(L)$, which is a vector bundle by Lemma 1.2. Obviously the composed morphism $P \rightarrow \pi^{*} P_{X}(L) \rightarrow \pi^{*} L$ is surjective. Let $Q_{0}$ be the kernel of $P \rightarrow \pi^{*} L$, which is a locally free subsheaf of $\pi^{*}\left(\Omega_{X} \otimes L\right)=\operatorname{Ker}\left(\pi^{*} P_{X}(L) \rightarrow \pi^{*} L\right)$. By the similar method to that in Case I, we can construct a locally free subsheaf $\tilde{\Omega}_{0}$ of $\pi^{*}\left(\Omega_{X} \otimes L\right)$ containing $Q_{0}$. Moreover we may assume that there exists an exacl sequence

$$
0 \longrightarrow \pi^{*}\left(\Omega_{1} \otimes L\right) \longrightarrow \widetilde{\Omega}_{0} \longrightarrow \Omega_{*}^{1} \longrightarrow 0
$$

where $\Omega_{1}^{*}=\pi^{*}\left(\left(\Omega_{X} / \Omega_{1}\right) \otimes L\right) \otimes \bar{F}_{1}$, and that the composed morphism $Q_{0} \hookrightarrow \widetilde{\Omega}_{0} \rightarrow \Omega_{1}^{*}$ is surjective. Let $Q_{1}=\operatorname{Ker}\left(Q_{0} \rightarrow \Omega_{1}^{*}\right)$, which is a locally free subsheal of $\pi^{*}\left(\Omega_{1} \otimes L\right)=\operatorname{Ker}\left(\widetilde{\Omega}_{0} \rightarrow \Omega_{1}^{*}\right)$. Again we can construct a locally free subsheaf $\widetilde{\Omega}_{1}$ of $\pi^{*}\left(\Omega_{1} \otimes L\right)$ containing $Q_{1}$ and an exact sequence

$$
0 \longrightarrow \pi^{*}\left(\Omega_{2} \otimes L\right) \longrightarrow \widetilde{\Omega}_{1} \longrightarrow \Omega_{2}^{*} \longrightarrow 0
$$

with $\Omega_{2}^{*}=\pi^{*}\left(\left(\Omega_{1} / \Omega_{2}\right) \otimes L\right) \otimes \bar{F}_{2}$, such that the composed morphism $Q_{1} \hookrightarrow \widetilde{\Omega}_{1} \rightarrow \Omega_{2}^{*}$ is surjective. Repeating this process we finally obtain exact sequences of vector bundles,

$$
\begin{aligned}
& 0 \longrightarrow Q_{0} \longrightarrow P \longrightarrow \pi^{*} L \longrightarrow 0 \\
& 0 \longrightarrow Q_{i+1} \longrightarrow Q_{i} \longrightarrow \Omega_{i+1}^{*} \longrightarrow 0 \text { for } \quad 0 \leqq i \leqq r-1
\end{aligned}
$$

where $\Omega_{i}^{*}=\pi^{*}\left(\left(\Omega_{i-1} / \Omega_{i}\right) \otimes L\right) \otimes \bar{F}_{i}$.

Consequently in the Chow ring $A^{\cdot} \bar{X}$, we have

$$
c(P)=\left\{1+\pi^{*} c_{1}(L)\right\} \cdot \prod_{j=0}^{r-1}\left\{1+\pi^{*} c_{1}\left(\Omega_{j} / \Omega_{j+1}\right)+\pi^{*} c_{1}(L)+c_{1}\left(\bar{F}_{j+1}\right)\right\} .
$$

Of course, we need not assume that $\operatorname{rank}\left(\Omega_{j-1} / \Omega_{j}\right)=1$ if $F_{j-1}=F_{i}$, and we can 
formulate the theorem even if there exists a birational proper morphism $\rho: X^{\prime} \rightarrow X$ such that $\rho^{*} \Omega_{X}$ has such a filtration. We obtain the next theorem.

Theorem 2.3. Let $f: X \rightarrow \boldsymbol{P}^{n}$ be a generic immersion from a smooth variety' $X$ of dimension $r$ into a projective $n$-space and $\rho: X^{\prime} \rightarrow X$ be a birational proper morphism. Let

$$
0=\Omega_{p} \subset \Omega_{p-1} \subset \cdots \subset \Omega_{1} \subset \Omega_{0}=\rho^{*} \Omega_{X}
$$

be a filtration of $\rho^{*} \Omega_{X}$ by' subbundles. Let $F_{i}=F^{0}\left(\right.$ Coker $\left.\phi_{i}\right)$ i.e. the ideal generated by maximal minors of the local representation of $\phi_{i}$ by a matrix where $\phi_{i}$ denotes the composition of the canonical morphism $\rho^{*} f^{*} \Omega_{\boldsymbol{P} n} \rightarrow \rho^{*} \Omega_{X}$ with the projection $\rho^{*} \Omega_{X} \rightarrow \rho^{*} \Omega_{X} / \Omega_{i}$. Assume that $F_{i-1}=F_{i}$ if $\operatorname{rank}\left(\Omega_{i-1} / \Omega_{i}\right)$ $>1$. Let $\bar{\pi}: \bar{X} \rightarrow X^{\prime}$ be the successive blowing up associated with the sequence of ideals

$$
F_{1} \supseteqq F_{2} \supseteqq \cdots \supseteqq F_{p} .
$$

Then, every polar class $\left[M_{k}\right]$ in the Chow group A.X satisfies the equalities

$$
\begin{aligned}
{\left[M_{k}\right] } & =\pi_{*}\left(c_{k}(P) \cap[\bar{X}]\right) \quad \text { for } \quad k \geqq 0, \\
c(P) & =\Sigma c_{k}(P) \\
& =\pi^{*} c(L) \cdot \prod_{j=1}^{p}\left\{\bar{\pi}^{*} c\left(\left(\Omega_{j-1} / \Omega_{j}\right) \otimes \rho^{*} L\right)+c_{1}\left(\bar{F}_{j}\right)\right\},
\end{aligned}
$$

where $\bar{F}_{j}=F_{j} \mathcal{O}_{\bar{X}} \otimes\left(F_{j-1} \mathcal{O}_{\bar{X}}\right)^{-1}$ for $j \geqq 1 \quad$ and $\pi=\rho \bar{\pi}$.

\section{§3. Examples}

In this section we apply our theorems to several examples.

For a rational equivalence class $\alpha \in A_{0} X$ of 0 -cycles, we can define its degree $\int \alpha=s_{*} \alpha \in A_{0} \operatorname{Spec}(k) \cong \mathbf{Z}$, where $s: X \rightarrow \operatorname{Spec}(k)$ is the structure morphism. Let $f: X \rightarrow \boldsymbol{P}^{n}$ be a morphism and $L=f^{*} \mathcal{O}_{\boldsymbol{P} n}(1)$. For a class of $j$-cycles $\beta \in A_{j} X$, its degree is defined to be $\int c_{1}(L)^{j} \cap \beta$, where $c_{1}(L)$ denotes the first Chern class of $L$.

Our theorems yield also numerical equalities by comparing the degree of terms. In the following we will discuss not only the equalities among rational equivalence classes but also numerical equalities.

Example 1 (Eager, Todd). Let $X$ be an $r$-dimensional smooth subvariety of $\boldsymbol{P}^{n}$. By Theorem 2.2 and Theorem 2.3 the $k$-th polar class of $X$ is given by 


$$
\begin{aligned}
{\left[M_{k}\right] } & =c_{k}\left(P_{X}(L)\right) \cap[X]=\sum_{i=0}^{k}\left(\begin{array}{c}
r+1-k+i \\
i
\end{array}\right) c_{h-i}\left(\Omega_{X}\right) c_{1}(L)^{i} \cap[X] \\
& =s_{k}\left(N^{2} \otimes L^{-1}\right) \cap[X]
\end{aligned}
$$

where $N$ denotes the conormal bundle of $X$ in $\mathbb{P}^{n}$. Here the $k$-th Segre class $s_{k}\left(N^{2} \otimes L^{-1}\right)$ is defined by the equality

$$
s\left(N^{2} \otimes L^{-1}\right)=\sum_{h=0}^{r} s_{k}\left(N^{2} \otimes L^{-1}\right)=c(N \otimes L)^{-1} .
$$

Before giving further examples, we will make a short remark about the meaning of terms involving the Chern class of the blown-up locally principal ideal.

Let $q: X^{\prime} \rightarrow X$ be the blowing up along a subvariety $V$ defined by a sheaf of ideals $I$. Let $D$ denote the exceptional divisor of $q$. Let $\alpha: V \rightarrow X$ and $\beta: D \rightarrow X^{\prime}$ be the inclusion morphisms, and $p: D \rightarrow V$ be the restriction of $q$ to $D$. Set $I^{\prime}=I \mathcal{O}_{X^{\prime}}$, which is a locally principal sheaf of ideals. We define

$$
s_{i}(V, X)=-q_{*}\left(c_{1}\left(I^{\prime}\right)^{r-i} \cap\left[X^{\prime}\right]\right) \in A_{i} X
$$

for every $i$ with $0 \leqq i<r$. Then, by Kleiman [3], Chapter II, Section D, we know the following three facts.

(1) $s_{i}(v, X)=\alpha_{*} p_{*}\left(c_{1}\left(\mathcal{O}_{D}(1)\right)^{r-1-i} \cap[D]\right)$. Thus, $s_{i}(V, X)$ has a support in $V$.

(2) $s(V, X)=\sum_{i} s_{i}(V, X)=\sum_{i} e_{i}\left[V_{i}\right]+v$, where $V_{1}, V_{2}, \cdots$ denote the irreducible components of $V, e_{i}$ denotes the multiplicity of $V$ in $X$ at $V_{i}$, and $v$ is the class of a cycle involving no $\left[V_{i}\right]$.

(3) If $q^{\prime}: X^{\prime \prime} \rightarrow X$ is any proper birational morphism such that $I^{\prime \prime}=I \mathcal{O}_{X^{\prime \prime}}$ is locally principal, then $s_{i}(V, C)=-q_{*}^{\prime}\left(c_{1}\left(I^{\prime \prime}\right)^{r-i} \cap\left[X^{\prime \prime}\right]\right)$ for every $i$. Namely " $s(V, X)$ does not depend on the choice of $q . "$

Example 2 (Plücker formulae for plane curves). Let $X$ be an irreducible curve of degree $d$ in $\boldsymbol{P}^{2}, \pi: Z \rightarrow X \hookrightarrow \mathbb{P}^{2}$ be its normalization. Let $\pi_{1}: \bar{X} \rightarrow X$ denote the blowing up of the Jacobian ideal $J$ of $X$, and $I$ denote the ramification ideal with respect to $\pi$. By the above remark, we know

$$
\begin{aligned}
& -\pi_{1 *}\left(c_{1}\left(J \mathcal{O}_{\bar{X}}\right) \cap[\bar{X}]\right)=\sum_{x \in \operatorname{Sing} X} e_{x}[x], \\
& -c_{1}(I) \cap[Z]=\sum_{y \in \operatorname{Ram} f} \kappa_{y}[y] .
\end{aligned}
$$

Here $e_{x}$ (resp. $\kappa_{y}$ ) denotes the multiplicity of the Jacobian ideal $J$ at $x$. (resp. the ramification ideal $I$ at $y$.) Since the conormal bundle of $X$ is isomorphic to 
$L^{-d}$, applying Theorem 2.2, we have

$$
\begin{aligned}
& {\left[M_{0}\right]+\left[M_{1}\right]=\pi_{1 *}(c(P) \cap[X]),} \\
& c(P)=\left(1+\pi_{1}^{*} c_{1}\left(L^{-(d-1)}\right)-c_{1}\left(J \mathcal{O}_{\bar{X}}\right)\right)^{-1} \\
& \quad=1+(d-1) \pi_{1}^{*} c_{1}(L)+c_{1}\left(J \mathcal{O}_{\bar{X}}\right),
\end{aligned}
$$

where $\left[M_{k}\right]$ denotes the $k$-th polar locus of $X$. Consequently we have

$$
\begin{aligned}
& {\left[M_{0}\right]=[X]} \\
& {\left[M_{1}\right]=(d-1) c_{1}(L) \cap[X]-\sum_{x \in \operatorname{Sing} X} e_{x}[x] .}
\end{aligned}
$$

Let $\left[M_{k}^{\prime}\right]$ denote the $k$-th polar locus of $Z$. In turn applying Theorem 2.3, we have

$$
\begin{aligned}
& {\left[M_{0}^{\prime}\right]+\left[M_{1}^{\prime}\right]=c\left(P^{\prime}\right) \cap[Z]} \\
& \begin{aligned}
c\left(P^{\prime}\right) & =\left(1+\pi^{*} c_{1}(L)\right)\left(1+c_{1}\left(\Omega_{Z}\right)+\pi^{*} c_{1}(L)+c_{1}(I)\right) \\
& =1+c_{1}\left(\Omega_{Z}\right)+2 \pi^{*} c_{1}(L)+c_{1}(I) .
\end{aligned}
\end{aligned}
$$

Therefore

$$
\begin{aligned}
& {\left[M_{0}^{\prime}\right]=[Z],} \\
& {\left[M_{1}^{\prime}\right]=c_{1}\left(\Omega_{Z}\right) \cap[Z]+2 \pi^{*} c_{1}(L) \cap[Z]-\sum_{y \in \operatorname{Ram} f} \kappa_{y}[y] .}
\end{aligned}
$$

Moreover we obtain numerical formulae for $c=\operatorname{deg}\left[M_{1}\right]=\int\left[M_{1}\right]$

$$
\begin{aligned}
& c=d(d-1)-\sum_{x \in \operatorname{Sing} X} e_{x}, \\
& c=2(g-1)+2 d-\sum_{y \in \operatorname{Ram} f} \kappa_{y},
\end{aligned}
$$

because $\pi_{*}\left[M_{k}^{\prime}\right]=\left[M_{k}\right]$ by definition (see Piene [5] or Urabe [8]) and $\int c_{1}(L) \cap[X]=d$. Of course, $g$ denotes the genus of $X$.

These are nothing but the classical Plücker formulae.

Example 3 (Piene). Let $X \subset \mathbb{P}^{n}$ be a local complete intersection of dimension $r, N$ be its conormal bundle. By Hironaka-Kleiman's Theorem we can choose a birational proper morphism $\rho: X^{\prime} \rightarrow X$ such that $\rho^{*} N$ is a successive extension by line bundles. Let $0=N_{0} \subset N_{1} \subset \cdots \subset N_{n-r}=\rho^{*} N$ be the associated filtration. Applying Theorem 2.2, we have

$$
\begin{aligned}
c_{1}(P) & =\sum_{i=1}^{n-r}\left\{-\pi^{*} c_{1}\left(N_{i}\right)+\pi^{*} c_{1}\left(N_{i-1}\right)-\pi^{*} c_{1}(L)+c_{i}\left(\bar{F}_{i}\right)\right\} \\
& =-\pi^{*} c_{1}(N)-(n-r) \pi^{*} c_{1}(L)+c_{1}\left(J \mathcal{O}_{\bar{X}}\right)
\end{aligned}
$$

and

$$
\left[M_{1}\right]=\left(c_{1}\left(N^{2}\right)-(n-r) c_{1}(L)\right) \cap[X]-\sum e_{\alpha}\left[S_{\alpha}\right]
$$


which coincides with Proposition 2.1 in Piene [5]. Here, $S_{1}, S_{2}, \ldots$ are integral romponents of codimension 1 of the singular locus and $e_{\alpha}$ denotes the multiplicity of the Jacobian ideal $J$ at the generic point of $S_{\alpha}$.

Example 4 (Pohl, Piene). Let $X$ be a smooth variety of dimension $r$, $f: X \rightarrow \mathbb{P}^{n}$ be a generic immersion. We can choose a birational proper morphism $\rho: X^{\prime} \rightarrow X$ such that $\rho^{*} \Omega_{X}$ is a successive extension by line bundles. Applying Theorem 2.3, we have

$$
c_{1}(P)=\pi^{*} c_{1}\left(\Omega_{X}\right)+(r+1) \pi^{*} c_{1}(L)+c_{1}\left(I \mathcal{O}_{\bar{X}}\right)
$$

and

$$
\left[M_{1}\right]=\left(c_{1}\left(\Omega_{X}\right)+(r+1) c_{1}(L)\right) \cap[X]-\sum \kappa_{\beta}\left[R_{\beta}\right]
$$

where $R_{1}, R_{2}, \ldots$ are the integral components of codimension 1 of the remification locus and $\kappa_{\beta}$ is the multiplicity of the ideal $F^{0}\left(\Omega_{f}\right)$ at the generic point of $R_{\beta}$. This coincides Proposition 2.4 in Piene [5], of course.

Example 5. Let $X \subset \mathbb{P}^{n}$ be a complete intersection of degree $\left(d_{1}, d_{2}\right.$, $\left.\ldots, d_{n-r}\right)$ i.e. $X=\cap{ }_{i=1}^{n-r} V_{+}\left(F_{i}\right)$ where $F_{i}$ is a homogeneous polynomial of degree $d_{i}$.

For every integer $k$ with $0 \leqq k \leqq n-r$, let $J_{h}$ be an ideal in $\mathcal{O}_{X}$ generated by

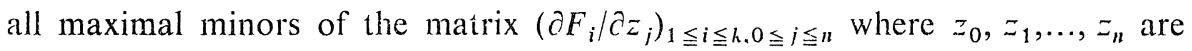
the homogeneous coordinates of $\mathbb{P}^{n}$ and homogeneous elements are regarded as ones in $\mathcal{O}_{X}$ after substituting homogeneous coordinates for inhomogeneous ones. The conormal bundle of $X$ is isomorphic to $\bigoplus_{l=1}^{n-r} L^{-d_{i}}$ and it is a successive extension by line bundles. It is easy to see that the sequence of ideals defined in Theorem 2.2 is equal to

$$
J_{1} \supseteqq J_{2} \supseteqq \cdots \supseteqq J_{n-r}
$$

in this case.

Let $\pi: \bar{X} \rightarrow X$ be the successive blowing up associated with (J). Applying Theorem 2.2, we obtain an explicit formula for $c(P)$ :

$$
c(P)=\prod_{i=1}^{n-r}\left\{1-\left(d_{i}-1\right) \pi^{*} c_{1}(L)+c_{1}\left(J_{i-1} \mathcal{O}_{\bar{X}}\right)-c_{1}\left(J_{i} \mathcal{O}_{\bar{X}}\right)\right\}^{-1} .
$$

Next we write down the explicit formula for every polar class $\left[M_{k}\right]$ in the case where $r=2$ and $n=4$. As for the case where $r=2$ and $n=3$, it has already been computed in Piene [5] (Theorem 2.3, Corollary 3.7, Example 3.11). Carrying out the calculation, we obtain the following for $X=V_{+}\left(F_{1}\right) \cap V_{+}\left(F_{2}\right) \subset \mathbb{P}^{4}$ with 
$d_{i}=\operatorname{deg} F_{i}(i=1,2)$.

$$
\begin{aligned}
c_{0}(P)= & 1 \\
c_{1}(P)= & \left(d_{1}+d_{2}-2\right) \pi^{*} c_{1}(L)+c_{1}\left(J_{2} \mathcal{O}_{\bar{X}}\right), \\
c_{2}(P)= & \left\{\left(d_{1}-1\right)^{2}+\left(d_{1}-1\right)\left(d_{2}-1\right)+\left(d_{2}-1\right)^{2}\right\} \pi^{*} c_{1}(L)^{2} \\
& +\left(d_{1}-d_{2}\right) \pi^{*} c_{1}(L) c_{1}\left(J_{1} \mathcal{O}_{X}\right) \\
& +\left(d_{1}+2 d_{2}-3\right) \pi^{*} c_{1}(L) c_{1}\left(J_{2} \mathcal{O}_{X}\right) \\
& +c_{1}\left(J_{1} \mathcal{O}_{X}\right)^{2}-c_{1}\left(J_{1} \mathcal{O}_{\bar{X}}\right) c_{1}\left(J_{2} \mathcal{O}_{\bar{X}}\right)+c_{1}\left(J_{2} \mathcal{O}_{\bar{X}}\right)^{2}
\end{aligned}
$$

Let $S_{1}, S_{2}, \ldots, S_{p}$ be the set of integral components of codimension 1 of the singular locus i.e. $\operatorname{Spec}\left(\mathcal{O}_{\bar{X}} / J_{2}\right)$ and for an integer $q$ with $q \leqq p$ we assume that the set of integral components of codimension 1 of $\operatorname{Spec}\left(\mathcal{O}_{\bar{X}} / J_{1}\right)$ consists of $S_{1}, \ldots, S_{q}$. Let $e_{\alpha}$ (resp. $f_{\alpha}$ ) denote the multiplicity of $J_{1}$ (resp. $J_{2}$ ) at the generic point of $S_{\alpha}$. We then have

$$
\begin{aligned}
& {\left[M_{0}\right]=[X]} \\
& \begin{aligned}
{\left[M_{1}\right]=} & \left(d_{1}+d_{2}-2\right) c_{1}(L) \cap[X]-\sum_{\alpha=1}^{p} e_{\alpha}\left[S_{\alpha}\right], \\
{\left[M_{2}\right]=\left\{\left(d_{1}-1\right)^{2}+\left(d_{1}-1\right)\left(d_{2}-1\right)+\left(d_{2}-1\right)^{2}\right\} c_{1}(L)^{2} \cap[X] } & \\
& -\left(d_{1}-d_{2}\right) \sum_{\alpha=1}^{p} e_{\alpha} c_{1}(L) \cap\left[S_{\alpha}\right] \\
& -\left(d_{1}+2 d_{2}-3\right) \sum_{\beta=1}^{q} f_{\beta} c_{1}(L) \cap\left[S_{\beta}\right] \\
& -v,
\end{aligned}
\end{aligned}
$$

where

$$
v=-\pi_{*}\left\{\left(c_{1}\left(J_{1} \mathcal{O}_{\bar{X}}\right)^{2}-c_{1}\left(J_{1} \mathcal{O}_{\bar{X}}\right) c_{1}\left(J_{2} \mathcal{O}_{\bar{X}}\right)+c_{1}\left(J_{2} \theta_{\bar{X}}\right)^{2}\right) \cap[X]\right\} .
$$

If we assume moreover that $V_{+}\left(F_{1}\right)$ has no singular point on $X$ and every singular point of $X$ is isolated, then we have

$$
\begin{aligned}
& \mu_{2}=\operatorname{deg}\left[M_{2}\right] \\
& \quad=\left\{\left(d_{1}-1\right)^{2}+\left(d_{1}-1\right)\left(d_{2}-1\right)+\left(d_{2}-1\right)^{2}\right\} d_{1} d_{2}-\sum_{x \in \operatorname{Sing} X} e_{x},
\end{aligned}
$$

where $e_{x}$ denotes the multiplicity of the Jacobian ideal $J=J_{2}$ at the singular point $x \in \operatorname{Sing} X$, because by assumption $c_{1}\left(J_{1} \mathcal{O}_{\bar{X}}\right)=c_{1}\left(\mathcal{O}_{\bar{X}}\right)=0$ and $-\pi_{*}\left(c_{1}\left(J_{2} \mathcal{O}_{X}\right)^{2} \cap[\bar{X}]\right)=\sum_{x \in \operatorname{Sing} X} e_{x}[x]$.

Example 6 (Ruled surfaces). Let $X$ be a ruled surface of genus $p$. Namely $X$ is the bundle $X=\boldsymbol{P}(E)$ of projective lines over a smooth projective curve $C$ of genus $p$ associated to a locally free sheaf $E$ of rank 2 on $C$. We denote by $g$ 
the projection $g: X \rightarrow C$. Let $f: X \rightarrow \boldsymbol{P}^{3}$ be a birational morphism which carries the fibres of $X$ onto lines in $\boldsymbol{P}^{3}$. The image $f(X)$ is also called a ruled surface. The cotangent bundle $\Omega_{X}$ has a filtration $g^{*} \Omega_{0} \hookrightarrow \Omega_{X}$. As the restriction of $f$ to every fibre of $X$ is a linear isomorphism, we know $F_{1}=F^{0}\left(\operatorname{Coker}\left(f^{*} \Omega_{p^{4}} \rightarrow\right.\right.$ $\left.\left.\Omega_{X} / g^{*} \Omega_{C}\right)\right)=\mathcal{O}_{X}$.

Applying Theorem 2.3, we have

$$
\begin{array}{r}
c(P)=\left(1+\pi^{*} c_{1}(L)\right)\left(1+\pi^{*} c_{1}\left(\Omega_{g}\right)+\pi^{*} c_{1}(L)\right) \\
\otimes\left(1+\pi^{*} g^{*} c_{1}\left(\Omega_{C}\right)+\pi^{*} c_{1}(L)+c_{1}\left(I \mathcal{O}_{X}\right)\right)
\end{array}
$$

where $\pi: \bar{X} \rightarrow X$ is the blowing up with the center of the ramification ideal $I$ with respect to $f$, and $L=f^{*} \mathcal{O}_{P^{3}}(1)$.

Replacing the bundle $E$ by $E \otimes N$ where $N$ is some invertible sheaf on $C$ if necessary, we may assume $\mathcal{O}_{X}(1)=f^{*} \mathcal{O}_{\mathbb{P}^{3}}(1)$. Set $h=f^{*} c_{1}\left(\mathcal{O}_{\mathbb{P}^{3}}(1)\right)$ $=c_{1}\left(\mathcal{O}_{X}(1)\right), e=g^{*} c_{1}(E), \gamma=g^{*} c_{1}\left(\Omega_{C}\right)$ and $i=c_{1}\left(I \mathcal{O}_{X}\right)$. We know $c_{1}\left(\Omega_{g}\right)=e-2 h$ because of the exact sequence $0 \rightarrow \Omega_{g} \rightarrow g^{*} E(-1) \rightarrow \mathcal{O}_{X} \rightarrow 0$. Moreover $e^{2}=0$, $e \gamma=0, \gamma^{2}=0$ because $C$ is a curve and $g^{*}$ is multiplicative, and by the fundamental relation of Chern classes $h^{2}-e h=0$. Namely

$$
\begin{aligned}
c(P) & =\left(1+\pi^{*} h\right)\left(1+\pi^{*}(e-h)\right)\left(1+\pi^{*}(\gamma+h)+i\right) \\
& =1+\pi^{*}(e+\gamma+h)+i+\pi^{*} h^{2}+\pi^{*} e \cdot i .
\end{aligned}
$$

We have consequently

$$
\begin{aligned}
& {\left[M_{1}\right]=\left(g^{*} c_{1}(E)+g^{*} c_{1}\left(\Omega_{C}\right)+c_{1}(L)\right) \cap[X]-\sum e_{\alpha}\left[R_{\alpha}\right],} \\
& {\left[M_{2}\right]=c_{1}(L)^{2} \cap[X]-\sum e_{\alpha} g^{*} c_{1}(E) \cap\left[R_{\alpha}\right],}
\end{aligned}
$$

where $R_{1}, R_{2}, \ldots$ are the integral components of the ramification locus of codimension 1 and $e_{\alpha}$ denotes the multiplicity of the ramification ideal $I$ at the generic point of $R_{\alpha}$.

\section{References}

[1] Borel, A. and Serre, J.-P., Le théoréme de Riemann-Roch, Bull Soc. math. France, 86 (1958), 97-136.

[2] Fulton, W., Rational equivalence for singular varieties, Publ. Math. I.H.E.S., 45 (1975), 147-167.

[3] Kleiman, S. L., The enumerative theory of singularities, Real and Complex Singularities, Oslo 1976, Sijthoff and Noordhoff, 1977, 297-396.

[4] Piene, R., Numerical characters of a curve in projective $n$-space, ibid., 475-494.

[5] Polar classes of singular varieties, Ann. Scient. Éc. Norm. Sup. $4^{\mathrm{e}}$ série, 11 (1978), 247-276. 
[6] Pohl, W., Extrinsic complex projective geometry, Proceedings of the Conference on Complex Analysis, Mineapolis 1964, Springer-Verlag, 1965, 18-29.

[7] Porteous, I. R., Todd's canonical classes, Liverpool singularities symposium I, Lecture notes in math., 192, Springer-Verlag, 1971, 308-312.

[8] Urabe, T., Duality of numerical characters of polar loci, Publ. RIMS, Kyoto Univ., 17 (1981), 331-345. 